\title{
The preparation of 3,5-dihydroxy-4- isopropylstilbene nanoemulsion and in vitro release
}

This article was published in the following Dove Press journal:

International Journal of Nanomedicine

31 March 201 I

Number of times this article has been viewed

\author{
Yue Zhang ${ }^{1,2}$ \\ Jungang Gao' \\ Hetang Zheng ${ }^{2}$ \\ Ran Zhang ${ }^{3}$ \\ Yucui Han ${ }^{2}$ \\ 'College of Chemistry and \\ Enviromental Science, Hebei \\ University, Baoding, China; ${ }^{2}$ School \\ of Chemical and Pharmaceutical \\ Engineering, Hebei University \\ of Science and Technology, \\ Shijiazhuang, China; ${ }^{3}$ College of \\ Chemical Engineering, East China \\ University of Science and Technology, \\ Shanghai, China
}

Abstract: We have reported a novel procedure to prepare 3,5-dihydroxy-4-isopropylstilbene (DHPS) nanoemulsion, using a low-energy emulsification method. Based on the phase diagram, the optimum prescription of nanoemulsion preparation was screened. With polyoxyethylenated castor oil (EL-40) as the surfactant, ethanol as the co-surfactant, and isopropyl myristate (IPM) as the oil phase, the DHPS nanoemulsion was obtained with a transparent appearance, little viscosity, and spherically uniform distribution verified by transmission electron microscopy and laser scattering analyzer. The nanoemulsion was also determined by FT-Raman spectroscopy. The DHPS nanoemulsion demonstrated good stability and stable physical and chemical properties. The nanoemulsion dramatically improved the transdermal release of DHPS (from $8.02 \mu \mathrm{g} \cdot \mathrm{cm}^{-2}$ to $273.15 \mu \mathrm{g} \cdot \mathrm{cm}^{-2}$ ) and could become a favorable new dosage form for DHPS.

Keywords: nanoemulsion, 3,5-dihydroxy-4-isopropylstilbene, DHPS, pseudo-ternary phase diagram

\section{Introduction}

3,5-Dihydroxy-4-isopropylstilbene (DHPS) (also named 2-isopropyl-5-(2phenylethenyl)-benzene-1,3-diol) belongs to the stilbene family and was first identified as a bacterial metabolite of the antimicrobial compound. ${ }^{1-5}$ This compound has attracted much attention for its diversified pharmacological activities including anti-inflammation and marked effect on T lymphocytes, mast cells, neutrophils, and macrophages ${ }^{6,7}$ After the rapid progress in pharmacological studies, DHPS has been proposed to serve as an effective drug for many diseases such as inflammatory bowel disease, rheumatoid arthritis, and autoimmune skin disorders. At the beginning of the twenty-first century, DHPS (numbered WBI-2001) was developed by Welichem Biotech Inc. (Burnaby, BC) as a new drug for atopic dermatitis and psoriasis, which are chronic skin diseases caused by a disorder of the autoimmune system. ${ }^{8,9}$ In the prior clinical trials in Canada, DHPS was shown to be effective and safe. ${ }^{10}$ In 2009, Celestial Pharmaceuticals (Shenzhen) Co Ltd (China) had also gained approval from the State Food and Drug Administration (SFDA) for the clinical trials of DHPS (Benvitimod) in China. However, the drug's instability and insolubility in water resulted in the alteration of properties and poor skin permeability. Therefore, the drug had no curative effect. To overcome the problem, it is necessary to seek a new vehicle to elevate the stability and solubility of DHPS in water and promote skin permeability.

Nanoemulsion is a new drug delivery system possessing isotropic, optical transparency or translucence characteristics and of special concern for practical applications. ${ }^{1{ }^{11}, 12}$ Nanoemulsions can be applied in the pharmaceutical field as drug delivery systems 
not only for oral administration, ${ }^{13,14}$ but also for parenteral, ${ }^{15}$ or transdermal administration, ${ }^{16}$ especially ocular. ${ }^{17}$ The drug is placed in the center of a small nanoemulsion droplet, $30-300 \mathrm{~nm}$, and is protected from oxidation, hydrolysis, and volatilization. Nanoemulsions have been reported to extend the drug response time and improve the efficacy of poorly water-soluble drugs as well as bioavailability. ${ }^{18-20}$ To date, compared with the oral carriers, few studies on nanoemulsion as topical carriers have been published. But it is still expected to be applied in topical delivery, taking advantage of good solubility, low skin irritation, and great permeability. ${ }^{21}$ In this work, we aimed to establish an oil-in-water $(\mathrm{O} / \mathrm{W})$ nanoemulsion delivery system for transdermal administration, in which DHPS was incorporated into the nanoemulsion concentrate so that the solubility and skin permeability of DHPS, and its therapeutic efficacy, could be dramatically enhanced. ${ }^{16,22,23}$ Literature reviews have so far revealed no studies on the nanoemulsion as a delivery system for DHPS, including the preparation and release study of DHPS nanoemulsion. In this study, we prepared and characterized DHPS nanoemulsion, and investigated its transdermal in vitro release and its effect as an active drug on the stabilities of the concentrate and corresponding nanoemulsion.

\section{Methods}

\section{Materials}

DHPS (structure shown in Scheme 1) was synthesized in the laboratory, ${ }^{24}$ and isopropyl myristate (IPM) was bought from Shanghai LEASUN Chemical Co. Ltd (Shanghai, China). Various surfactants such as polyoxyethylene sorbitan fatty acid esters (Tween-80) and polyoxyethylenated castor oil (EL-40) were purchased from Tianjin Yongda Chemical Reagent Development Center (Tianjin, China). Methanol was chromatographically pure and purchased from Kangkede (Tianjin, China). Other chemical reagents were all analytically pure grades and purchased from Shijiazhuang Modern Reagent Co (Shijiazhuang, China). Soy bean salad oil, olive oil, and peanut oil were all of food grade and purchased from COFCO (Tianjin, China). Water was twice distilled.

\section{Characterization analyses}

The process of identifying the structural type of the nanoemulsion via staining is as follows: the water-soluble methylthionine chloride and oil-soluble Sudan red were added to the same batch of the nanoemulsion. The diffusion rate of two kinds of staining agents in the nanoemulsion was recorded. The transmission electron microscopy (TEM, H-7650; Hitachi, Tokyo, Japan) was used to monitor the morphology

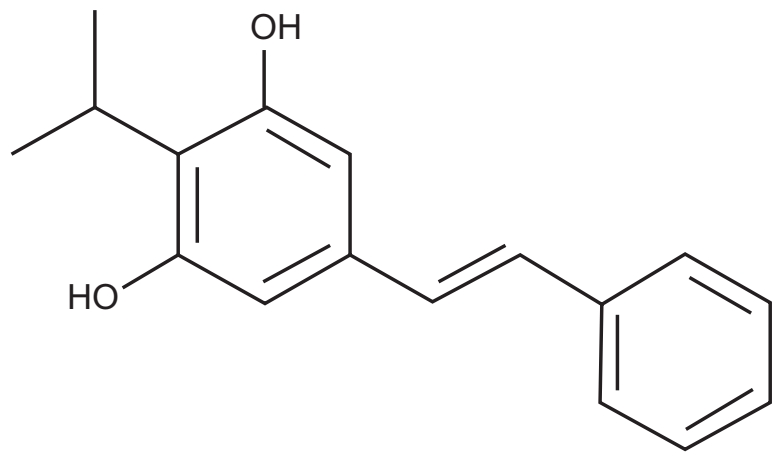

Scheme I Structure of DHPS.

and distribution of nanoemulsion droplets and FT-Raman spectroscopy (MultiRam; Bruker, Madison, WI) was used to determine the components. The mean size of nanoemulsion droplets was detected by Laser Scattering Analyzer (NanoS90; Malvern Instruments, Malvern, Worcestershire, UK). The amount of DHPS in the solubility and stability experiments, and in vitro transdermal release, was detected by highperformance liquid chromatography (HPLC, LC-10AT VP; Shimadzu, Kyoto, Japan). The mobile phase was a mixture of methanol and distilled water $(V / V=80 / 20)$. The column of HPLC was Phenomenexluna-C18 $(250 \times 4.6 \mathrm{~mm}, 5 \mu \mathrm{m})$. The flow rate was $1.0 \mathrm{~mL} /$ minute, the detection wavelength was $316 \mathrm{~nm}$, and the injected volume of the sample was $10 \mu \mathrm{L}$. The in vitro transdermal release experiment of DHPS nanoemulsion was carried out through rat skin in a Franz diffusion cell (RYJ-12B, Huanghai, China).

\section{Solubility of DHPS}

Achieving good solubility of drugs in the studied surfactants, co-surfactants, oil mediums, and their emulsifier is important in the development of nanoemulsions. EL-40 and Tween-80 were chosen as non-ionic surfactants, and ethanol, $i$-propanol, 1,2-propanediol, and $n$-butanol were used as short-chain cosurfactants. Frequently used liquid paraffin, IPM, soybean oil, and olive oil were used as oil mediums. The solubility performance of DHPS in the above components and their emulsifier were observed. Different proportions of the above components and DHPS were placed in small vials. The vials were vibrated in a water bath at $37^{\circ} \mathrm{C}$ for 24 hours, and then the solubilities of DHPS in different components were determined by HPLC.

\section{Phase diagrams}

The methods for the formation of nanoemulsions include highenergy and low-energy emulsification. Mechanical energy (such as high shear stirring, high-pressure homogenizers, and 
ultrasound generators) was utilized in a high-energy emulsifying process ${ }^{25}$ and chemical energy (potential energy) in the components was used in a low-energy emulsifying process. ${ }^{26}$ Compared with the high-energy formation of nanoemulsions, the low-energy emulsification method has a strong appeal in the development of a drug delivery system, which can take advantage of phase behavior, industial scale-up, reduced physical destruction of drugs, and the formation of smaller droplets. ${ }^{27-29}$ The low-energy methods are divided into phase inversion composition ${ }^{30}$ and phase inversion temperature (PIT) methods. ${ }^{31,32}$ Nanoemulsions prepared by phase inversion composition are strongly affected by compositions in the system and their instrinsic physicochemical properties. ${ }^{33}$ During the phase inversion composition emulsification process, as the components change (oil phase mixed with aqueous phase), the rapid transfer of hydrophilic materials between two phases is spontaneously generated. ${ }^{26}$ The interfacial area increased markedly, and a metastable emulsion state was generated. Therefore, the composition of components and their ratios strongly influence the formation of nanoemulsions and, simultaneously, their properties, such as the stability and distribution of particle size. ${ }^{34-36}$ For this reason, it is essential to establish a pseudoternary phase diagram in order to determine the existing region of the nanoemulsion. The phase diagrams were developed using the aqueous titration method with one axis representing the composition of SF and Co-SF, another representing water, and the third representing oil; the nanoemulsion region would be the basis for the selection of the formulation.

The emulsifiers and oil mediums were sealed in ampoules and vibro-mixed gently to be homogenized at $25^{\circ} \mathrm{C}$. Distilled water was then added continuously to the homogenized mixture. The phase inverse points and the mass ratio of all components corresponding to that point were recorded during the consecutive addition of distilled water, and then the boundary lines appeared. The isotropic liquid crystal line phase was identified by polarizing light microscopy (PLM, BX51; Olympus, Tokyo, Japan).

\section{Preparation of nanoemulsion}

A certain amount of DHPS was dissolved in co-surfactant at a constant temperature in a vial. The oil mediums, surfactants, and solution of co-surfactant with DHPS were thoroughly mixed. Distilled water was added continuously to the mixed liquid. As the amount of distilled water increased, the system suddenly became viscous, and liquid crystals appeared. The distilled water was added drop-wise until a stable, colorless, and transparent or translucent nanoemulsion was formed.

\section{Selection of surfactant and co-surfactant}

EL-40 and Tween-80 were chosen as non-ionic surfactants, and ethanol, $i$-propanol, 1,2-propanediol, and $n$-butanol were used as short-chain co-surfactants. IPM was used in the oil phase, according to oil solubility studies. The tests were designed under different $\mathrm{Km}$ (mass ratio of surfactant to cosurfactant) as 9:1, 8:2, 7:3, 6:4, 5:5, 4:6, 3:7, 2:8, 1:9, and the corresponding mass ratio of the combination of surfactant and co-surfactant (Smix) to IPM was varied as 1:9, 2:8, 3:7, 4:6, $5: 5,6: 4,7: 3,8: 2,9: 1$. The oil, surfactant, and co-surfactant of each group were well mixed to form an emulsion, and the total mass of all components was constant. Distilled water was added drop by drop into the emulsifier under ambient temperature. The emulsion appeared transparent and translucent, with low viscosity, and the Tyndall effect was observed. We noted that sometimes the system changed from clarity to turbidity, or from clarity to thickness, or back again from thickness to clarity, and noted down the quality percentage of all the components corresponding to the phase inversion point.

\section{Optimization of the formulation}

To obtain an effective emulsification, the surfactant and co-surfactant are usually combined. The mixing ratio of surfactant/co-surfactant, to form nanoemulsions, also needs careful consideration. Surfactant (EL-40) and co-surfactant (ethanol) were mixed in different mass ratios $(6: 1,4.5: 1,3: 1$, 1.5:1). For each group, IPM and the mixture of surfactantco-surfactant with specific $\mathrm{Km}$ were mixed well in a series of mass ratios ranging from 1:9 to 9:1.

\section{Stability study of DHPS nanoemulsion}

In order to avoid a metastable system, we performed various stability studies such as bearing centrifuging, heat, humidity, and light irradiating. The prepared formulations were sealed in the centrifuge tube and centrifuged at $10000 \mathrm{rpm}$ for 20 minutes. Phase separation (cracking or creaming) was observed. If DHPS nanoemulsion showed no instability during the centrifugation process, its stability was assessed according to the regulations of Chinese Pharmacopoeia ${ }^{37}$ including the high temperature test, high humidity test, and hard light irradiating test. The relative specifications of DHPS nanoemulsion were evaluated with sampling at 0,5 , and 10 days, respectively.

\section{In vitro transdermal release}

The abdominal skin of a mouse ( $25 \pm 2 \mathrm{~g}$, female, Kunming, China), which was depilated with $8 \%$ sodium sulfide and cleaned of fat, was washed with saline and then fixed to 


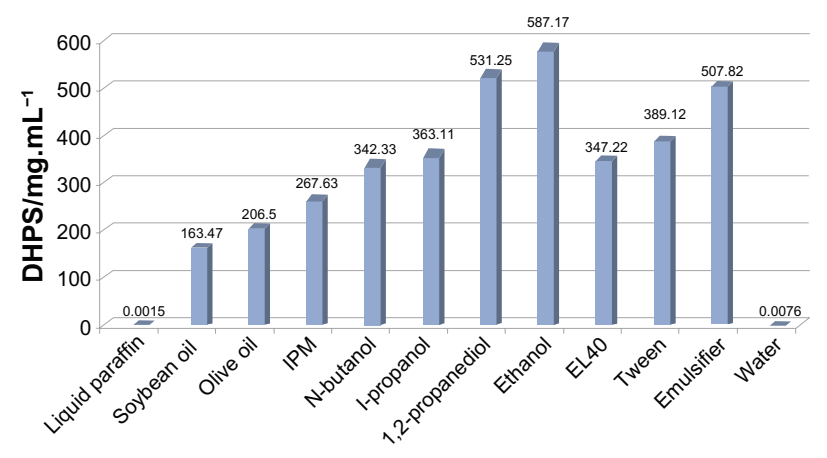

Figure I The solubility of DHPS in various components.

the Franz diffusion cell. The horny layer of skin faced the supply chamber, and $1.0 \mathrm{~g}$ DHPS nanoemulsion was added, in close contact with the skin. Sixteen milliliters of $0.9 \%$ sodium chloride solution $(M / M)$ was used as the receiving medium. The temperature of the receiving medium was kept at $37 \pm 0.5^{\circ} \mathrm{C}$ and the rotating speed was controlled at $120 \mathrm{rpm}$. At certain time intervals $(0.5,1,2,4,6,8,10$, and 12 hours), $5 \mathrm{~mL}$ receiving medium was taken out of the supply chamber and, simultaneously, an equal volume of fresh receiving medium was added to the supply chamber to keep the total volume unchanged. Released DHPS was quantified by HPLC and the cumulative release rate was calculated. During the in vitro transdermal experiment, the release behavior of the DHPS nanoemulsion was compared with that of the drug DHPS (20 mg DHPS suspended in the supply chamber). All the experiments were carried out in triplicate. The release behavior of DHPS nanoemulsion was compared with that of pure DHPS suspension. The accumulative osmotic quantity per unit area (Qt) can be described as:

$$
\mathrm{Qt}=\sum C i V i / A
$$

where $\mathrm{Ci}$ is the DHPS concentration in the tested samples, $\mathrm{Vi}$ is the volume of the receiving pool, and $\mathrm{A}$ is the effective area of the receiving pool.

\section{Results Solubility of DHPS}

As shown in Figure 1, DHPS is almost insoluble in distilled water and liquid paraffin, but has reasonable solubility in IPM, Tween-80, and EL-40. The solubility of the drug in the components is important for component screening, ${ }^{38}$ and therefore, IPM was selected as the oil phase, and Tween- 80 and EL-40 were chosen as the surfactants in the nanoemulsion formulation. The four co-surfactants all have favorable solubility for DHPS, although of the four co-surfactants, ethanol had the most advantages. The emulsifier formulations consisted of surfactant (EL-40), co-surfactant (ethanol), and drug dissolved in the oil (IPM) to provide a clear liquid at ambient temperature. The test results showed that the emulsifier had extremely good solvent properties for DHPS. The solubility of DHPS in the emulsifier was elevated to about 60,000 times that in water.

\section{Selection of surfactant and co-surfactant}

To select an ideal surfactant, the solubility of DHPS, the hydrophile-lipophile balance (HLB), and toxicity to the human body must be considered seriously. Based on solubility studies, both EL-40 and Tween-80 showed considerable solubility of DHPS, and appropriate HLB value (range 8 to 16). Because non-ionic surfactants facilitate the adjustment of the interfacial tension, they are appropriate candidates for favorable emulsifiers, as they exhibit low toxicity and play important roles in the drug delivery system. But as the results in Table 1 show, no phase change appears (no nanoemulsion was formed) when Tween- 80 was the surfactant, no matter which alcohol was used as co-surfactant, and no matter under which ratios. In contrast, the mixture of EL-40 with four types of co-surfactant in certain proportions facilitated the formation of nanoemulsions and, therefore, more choices were available for the selection of the co-surfactant formulation. In view of this investigation, the selection of EL-40 as the surfactant in the preparation of the DHPS nanoemulsion was appropriate.

Table I Impacts of surfactants and co-surfactants on the nanoemulsion formation

\begin{tabular}{|c|c|c|c|c|c|c|c|c|c|c|}
\hline \multirow[t]{2}{*}{ Surfactants } & \multirow[t]{2}{*}{ Co-surfactants } & \multicolumn{9}{|c|}{ Smix/oil } \\
\hline & & $9: 1$ & $8: 2$ & $7: 3$ & $6: 4$ & $5: 5$ & $4: 6$ & $3: 7$ & $2: 8$ & I:9 \\
\hline \multirow[t]{4}{*}{ EL-40 } & Ethanol & $\sqrt{ }$ & $\sqrt{ }$ & $\sqrt{ }$ & - & - & - & - & - & - \\
\hline & $n$-butanol & $\sqrt{ }$ & $\sqrt{ }$ & - & - & - & - & - & - & - \\
\hline & i-propanol & $\sqrt{ }$ & $\sqrt{ }$ & - & - & - & - & - & - & - \\
\hline & I,2-propanediol & $\sqrt{ }$ & $\sqrt{ }$ & $\sqrt{ }$ & - & - & - & - & - & - \\
\hline \multirow[t]{4}{*}{ Tween-80 } & Ethanol & - & - & - & - & - & - & - & - & - \\
\hline & n-butanol & - & - & - & - & - & - & - & - & - \\
\hline & i-propanol & - & - & - & - & - & - & - & - & - \\
\hline & I,2-propanediol & - & - & - & - & - & - & - & - & - \\
\hline
\end{tabular}

Notes: $\sqrt{ }$ represents the formation of nanoemulsion; - represents the absence of formation of nanoemulsion. 


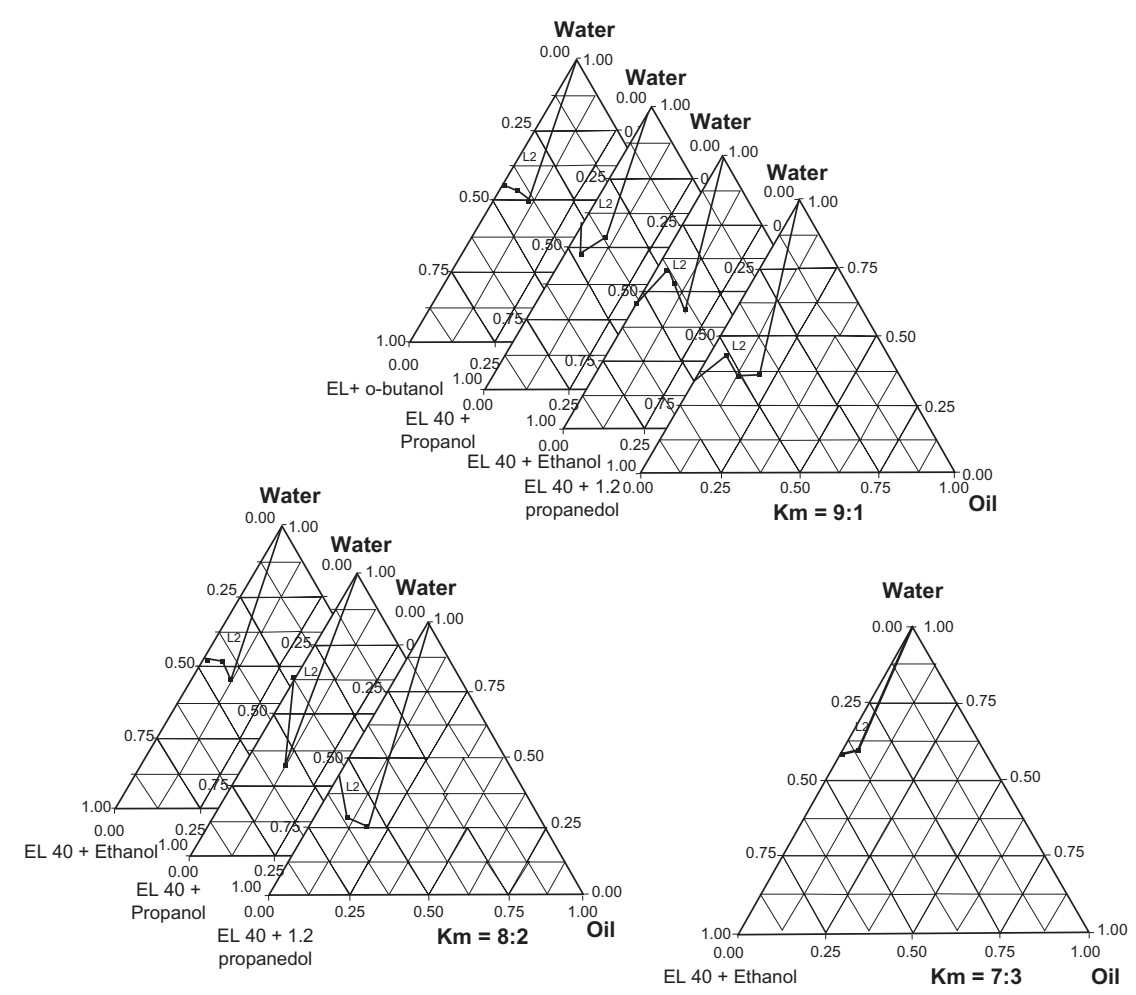

Figure 2 The ternary phase diagram under different conditions. Abbreviation: $L_{2}$, $\mathrm{O} / \mathrm{W}$ microemulsion area.
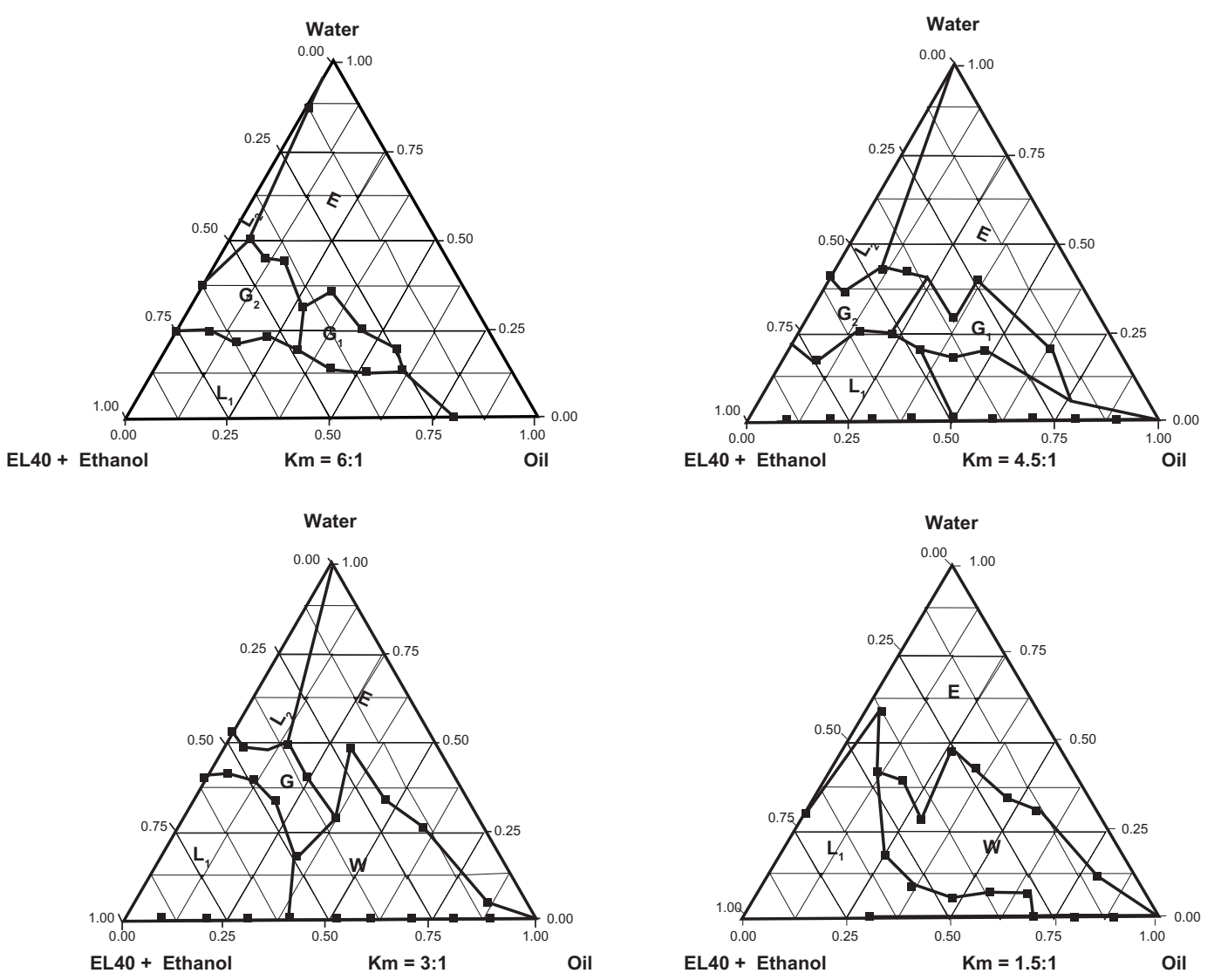

Figure 3 The ternary phase diagram under different proportion condition.

Abbreviations: $\mathrm{L}_{1}, \mathrm{~W} / \mathrm{O}$ nano-emulsion area; $\mathrm{L}_{2}, \mathrm{O} / \mathrm{W}$ nanoemulsion area; $\mathrm{G}$, viscous area; $\mathrm{G}_{1}$, viscous but opacified area; $\mathrm{G}_{2}$, viscous but clarified area; $\mathrm{E}$, ordinary emulsion area; $W$, the turbid area. 


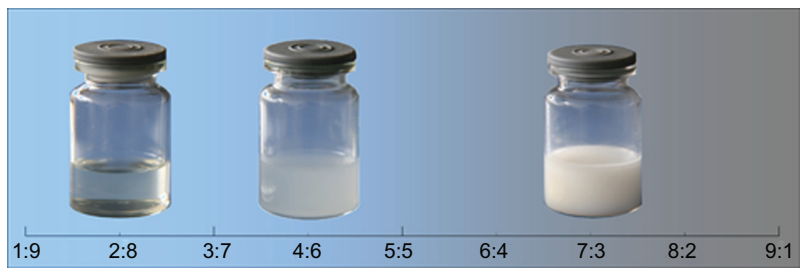

Figure 4 The phase behavior of nanoemulsions with different Smixs to oil.

A good co-surfactant can improve the dispersibility of droplets and drug absorption. ${ }^{39}$ EL-40 was combined with four different co-surfactants including $i$-propanol, 1,2propanediol, $n$-butanol and ethanol, in different mass ratios, respectively. The results are shown in Figure 2. All four co-surfactants can promote the formation of nanoemulsions when $\mathrm{Km}$ is taken as 9:1; the nanoemulsion region was the largest when 1,2-propanediol was used for the co-surfactant. No clear transitional phase inversion appears when $\mathrm{Km}$ is taken as 8:2 and $n$-butanol is taken as co-surfactant. At a $\mathrm{Km}$ of 7:3, only ethanol can arrange EL-40 in pairs to generate phase inversion. When $i$-propanol served as the co-surfactant, the area of nanoemulsion was relatively small and, in comparison, the areas of nanoemulsion in phase diagrams are larger when 1,2-propanediol or ethanol are used. Among these two co-surfactants, a larger amount of surfactants are required when 1,2-propanediol is used owing to its lower solubility of DHPS, which may be harmful to the skin. Therefore because ethanol is non-irritating, has gentle properties, no toxicity to the human body, and is the most common and readily available reagent, it was chosen to be the co-surfactant.

\section{Optimization of the formulation}

As Figure 3 shows, when $\mathrm{Km}=6: 1$, a small amount of oil $(5.2 \%)$ can be emulsified and the nanoemulsion region is faint. As the $\mathrm{Km}$ decreases, the nanoemulsion region increases, further increasing the proportion of co-surfactant $(\mathrm{Km}=3: 1)$; the nanoemulsion region increases to a large scale and a considerable amount of oil (15.6\%) can be emulsified. When $\mathrm{Km}$ decreases to 1.5:1, the co-surfactant occupies a bigger ratio and the nanoemulsion cannot be formed. Taking a comprehensive view of the nanoemulsion

Table 2 Physicochemical parameters of DHPS nanoemulsion $(n=3)$

\begin{tabular}{ll}
\hline Parameter & Results \\
\hline $\mathrm{pH}$ & $5.69 \pm 0.08$ \\
Refractive index & $1.3875 \pm 0.002$ \\
Viscosity/cP & $3.25 \pm 0.02$ \\
Structure type & $\mathrm{O} / \mathrm{W}$ \\
\hline
\end{tabular}

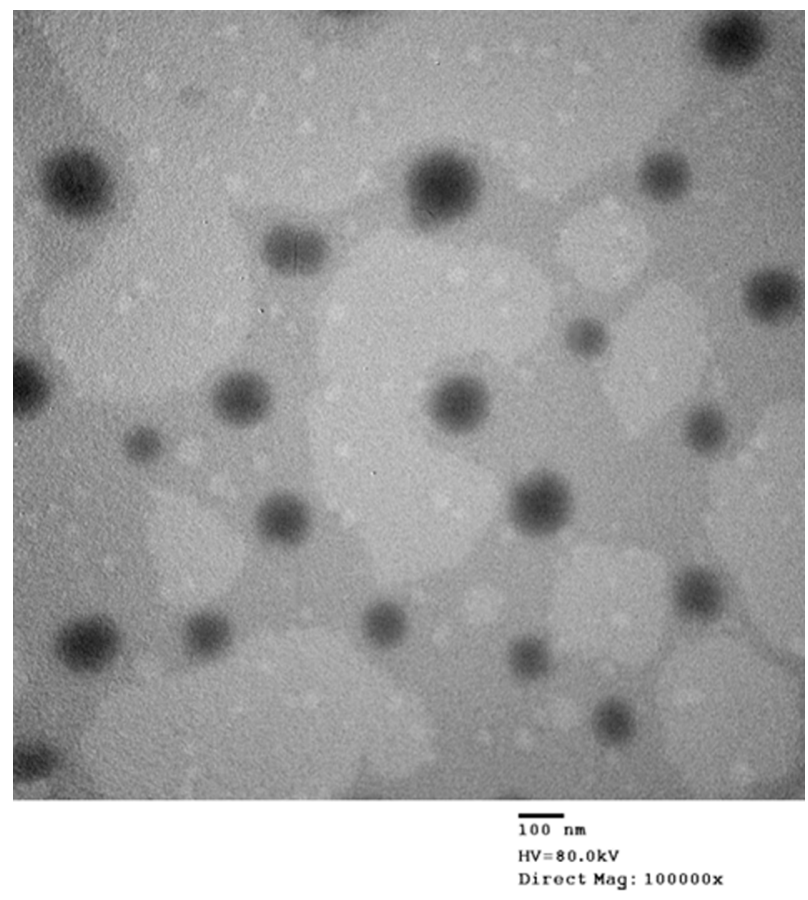

Figure $\mathbf{5}$ The TEM image of DHPS nanoemulsion particles.

region, and the lower amount of Smix (surfactant/ co-surfactant) used in this system, we finally selected the $\mathrm{Km}$ ratio as 3:1, and the ratio of Smix to oil as 3:7. Nanoemulsions prepared with this formulation were further studied for stability and potential clinical application. Under this optimized ratio, the prepared DHPS nanoemulsions with different mass ratios of Smix to oil were photographed and are shown in Figure 4.

\section{Characterization analyses}

Physicochemical properties of DHPS nanoemulsions

The $\mathrm{pH}$, viscosity, refractive index, and structure type of DHPS nanoemulsions are shown in Table 2.

\section{Morphology and size distribution of DHPS nanoemulsion}

TEM can directly produce images of targets with high resolution. The morphology and structure of the optimal

\section{Statistics graph (1 measurement)}

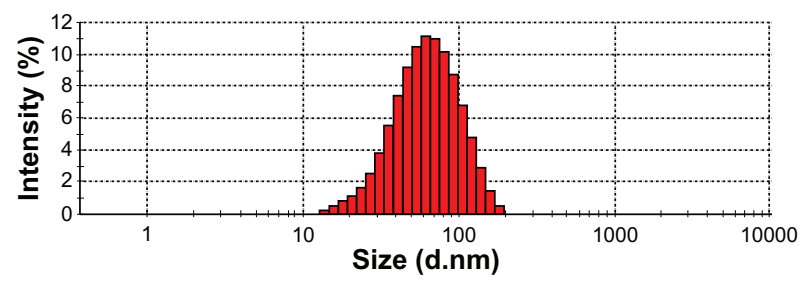

Figure 6 The mean size of DHPS nanoemulsion droplets. 


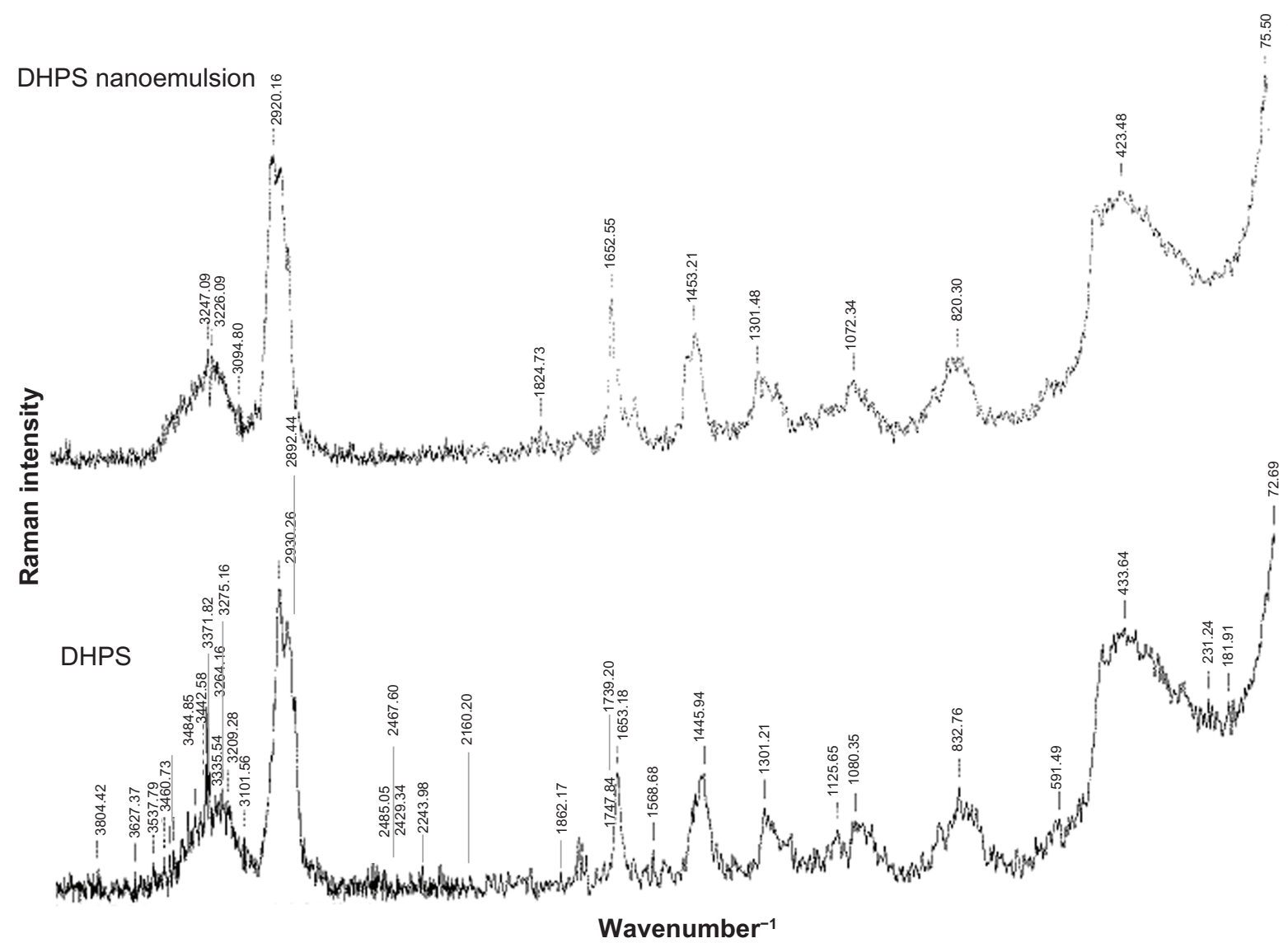

Figure 7 FT-Raman spectra.

nanoemulsion formulation were observed using TEM. As seen in Figure 5, the nanoemulsion droplet is round and has a smooth margin with a mean size of about $60.88 \mathrm{~nm}$ (Figure 6). The droplet size has a direct influence on the behavior of the nanoemulsion, especially the bioavailability of the delivered drug. In general, the smaller the droplet size in the nanoemulsion, the more favorable the expected result for in vivo application.

\section{Raman spectrum of DHPS nanoemulsion}

DHPS and the DHPS nanoemulsion were detected by FT-Raman spectroscopy at laser $350 \mathrm{~mW}$ scanning from $50 \mathrm{~cm}^{-1}$ to $4000 \mathrm{~cm}^{-1}$. Because the surfactants and co-surfactants have no clear characteristic scattering

Table 3 The stability of drug nanoemulsion $(n=3)$

\begin{tabular}{llll}
\hline Condition & pH & $\begin{array}{l}\text { Average } \\
\text { diameter/nm }\end{array}$ & Percentage/\% \\
\hline $0 \mathrm{~d}$ & $5.69 \pm 0.0 \mathrm{I}$ & $62.89 \pm 0.09$ & $99.78 \pm 0.0 \mathrm{I}$ \\
$60^{\circ} \mathrm{C}, 5 \mathrm{~d}$ & $5.67 \pm 0.0 \mathrm{I}$ & $63.19 \pm 0.10$ & $99.20 \pm 0.03$ \\
$60^{\circ} \mathrm{C}, 10 \mathrm{~d}$ & $5.64 \pm 0.0 \mathrm{I}$ & $62.0 \mathrm{I} \pm 0.08$ & $98.37 \pm 0.05$ \\
$4500 \pm 500 \mathrm{~lx}, 5 \mathrm{~d}$ & $5.66 \pm 0.0 \mathrm{I}$ & $63.12 \pm 0.1 \mathrm{I}$ & $98.95 \pm 0.02$ \\
$4500 \pm 500 \mathrm{~lx}, 10 \mathrm{~d}$ & $5.63 \pm 0.02$ & $63.32 \pm 0.12$ & $98.45 \pm 0.03$ \\
\hline
\end{tabular}

absorbance, DHPS and the DHPS nanoemulsion have almost the same FT-Raman spectra. In Figure 7, the scattering characteristic peaks at $1453 \mathrm{~cm}^{-1}$ are attributed to the conjugating aromatic rings in stilbene and there is an alkene scattering peak at $1652 \mathrm{~cm}^{-1}$. The peaks from $2920 \mathrm{~cm}^{-1}$ to $2930 \mathrm{~cm}^{-1}$ and the peak at $1301 \mathrm{~cm}^{-1}$ indicate the $i$-propyl group of DHPS.

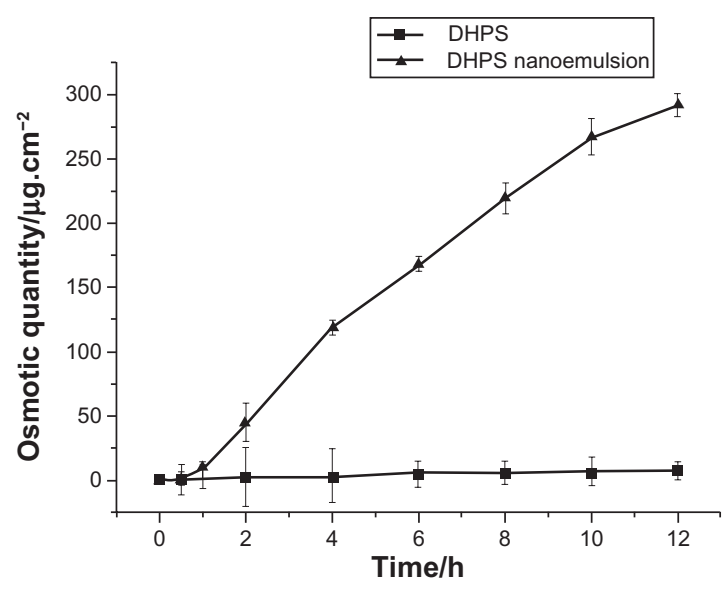

Figure 8 The in vitro transdermal release of drug DHPS and DHPS nanoemulsion $(n=3)$. 
Table 4 The accumulative osmotic quantity $(\mathrm{Qt})$ and the penetration rate $(\mathrm{J})$

\begin{tabular}{lll}
\hline Sample & Regression curve & $\boldsymbol{R}$ \\
\hline Drug DHPS & Qt $=0.53 \mathrm{t}+1.73$ & 0.9975 \\
DHPS nanoemulsion & $\mathrm{Qt}=24.5 \mathrm{It}-3.5 \mathrm{I}$ & 0.9914 \\
\hline
\end{tabular}

\section{Stability of the DHPS nanoemulsion}

After centrifuging, there was no sign of phase separation, creaming or cracking, and the nanoemulsion was still clear and transparent, with no turbidity and no crystal precipitation. The data in Table 3 show that there are no apparent changes in $\mathrm{pH}$, drop size, and concentration of nanoemulsion, which indicates good stability of the nanoemulsion loaded with DHPS.

\section{In vitro transdermal release}

In order to optimize drug delivery and achieve an ideal therapeutic effect, we have incorporated the drug into the heart of nanoemulsion droplets. Therefore, it was extremely important to observe the release of DHPS from the nanoemulsions in comparison with that of the drug. Because nanoemulsions provide better dispersion and larger contact surface area for drug molecules, an elevated solubility and permeability could be expected.

As seen from Figure 8, it is clear that DHPS released from nanoemulsion increases exponentially, while DHPS released from the drug suspension is rather low and almost constant. In a 12-hour period, the maximum accumulative osmotic quantity, Qt, of DHPS from the DHPS suspension is $8.02 \mu \mathrm{g} \cdot \mathrm{cm}^{-2}$, while the Qt of DHPS from DHPS nanoemulsion is up to $273.15 \mu \mathrm{g} \cdot \mathrm{cm}^{-2}$. During a defined time period, the transdermal release of DHPS from the suspension and the nanoemulsion follows the zero order kinetics equation, and the slope of the line indicates the penetration rate, $\mathrm{J}$ (Table 4). It is clear that the penetration rate, $\mathrm{J}$, of DHPS is $0.53 \mu \mathrm{g} \cdot \mathrm{cm}^{-2} \cdot \mathrm{h}^{-1}$ from the DHPS suspension, and $24.51 \mu \mathrm{g} \cdot \mathrm{cm}^{-2} \cdot \mathrm{h}^{-1}$ from the DHPS nanoemulsion. Therefore, the DHPS nanoemulsion has demonstrated significant transdermal advantages.

\section{Conclusion}

We have reported a novel procedure to prepare DHPS nanoemulsion and identified the optimal prescription through a number of experiments. With EL-40 as the surfactant, ethanol as the co-surfactant, and IPM as the oil phase, the DHPS nanoemulsion can be obtained with transparent appearance, low viscosity, and a spherically uniform distribution of droplets as verified by TEM, Raman spectroscopy, and laser scattering analyzer. For an optimal formulation, a mass ratio of EL-40 to ethanol $(\mathrm{Km})$ of $3: 1$ was chosen, and the Smix to oil was selected at 3:7. This optimized DHPS nanoemulsion has stable physical and chemical properties, and also good stability. The nanoemulsion has dramatically improved the DHPS transdermal effect, and the osmotic quantity of DHPS from the nanoemulsion formulations is $273.15 \mu \mathrm{g} \cdot \mathrm{cm}^{-2}$, significantly better than for the DHPS suspension $\left(8.02 \mu \mathrm{g} \cdot \mathrm{cm}^{-2}\right)$.

\section{Disclosure}

The authors declare no conflicts of interest in this work.

\section{References}

1. Hu KJ, Li JX, Wand WJ. Comparison of metabolites produced in vitro and in vivo by Photorhabdus luminescens, a bacterial symbiont of the entomopathogenic nematode Heterorhabditis megidis. Can J Microbiol. 1998;44:1072-1077.

2. Krow GR, Miles WH, Smiley PM, et al. Synthesis of antibiotic stilbenes using organomanganese arene complexes. J Org Chem. 1992;57:4040-4043.

3. Akhurst RJ. Antibiotic activity of Xenorhabdus spp., bacteria symbiotically associated with insect pathogenic nematodes of the families Heterorhabditidae and Steinernematidae. J Gen Microbiol. 1982;128:3061-3065.

4. Paul VJ, Frautschy S, Fenical W, et al. Antibiotics in microbial ecology: isolation and structure assignment of several new antibacterial compounds from the insectsymbiotic bacteria Xenorhabdus spp. J Chem Ecol. 1981;7:589-597.

5. Richardson WH, Schmidt TM, Nealson KH. Identification of an anthraquinone pigment and a hydroxystilbene antibiotic from Xenorhabdus luminescens. Appl Environ Microbiol. 1988;54:1602-1605.

6. Hu K, Webster JM. Antibiotic production in relation to bacterial growth and nematode development in Photorhabdus-Heterorhabditis infected Galleria mellonella larvae. FEMS Microbiology Lett. 2000; 189:219-223.

7. Tang LR, Chen GH, Li B, et al. Anti-inflammatory activities of stilbene analogs for targeting autoimmune diseases. Clin Immunol. 2007; 123:S124.

8. Chen GH, Li JX, Liu W, Webster JM, inventor; Welichem Biotech Inc., assignee. Novel bioactive diphenyl ethene compounds and their therapeutic applications. International Patent WO 2004031117. 2004 Apr 15.

9. Hu KJ, Li JX, Li B, et al. A novel antimicrobial epoxide isolated from larval Galleria mellonella infected by the nematode symbiont, Photorhabdus luminescens (Enterobacteriaceae). Bioorg Med Chem. 2006;14:4677-4681.

10. Chen GH, Webster JM. inventor; Welichem Biotech Inc., assignee. Anti-inflammatory and psoriasis treatment and protein kinase inhibition by hydroxystilbenes and novel stilbene derivatives and analogues. United States Patent US 20080255245. 2008 Oct 16.

11. Wang LJ, Dong JF, Chen J, et al. Design and optimization of a new self-nanoemulsifying drug delivery system. J Colloid Interface Sci. 2009;330:443-448.

12. Zhou HF, Yang Y, Liu GL. Preparation and Characterization of a lecithin nanoemulsion as a topical delivery system. Nanoscale Res Lett. 2010;5: 224-230.

13. Baluom M, Friedman DI, Rubinstein A. Absorption enhancement of calcitonin in the rat intestine by carbopol-containing submicron emulsions. Int J Pharm. 1997;154:235-243.

14. Vyas TK, Shahiwala A, Amiji MM. Improved oral bioavailability and brain transport of Saquinavir upon administration in novel nanoemulsion formulations. Int J Pharm. 2008;347:93-101. 
15. Muhannad J, Bernd WM. The effect of oil components and homogenization conditions on the physicochemical properties and stability of parenteral fat emulsions. Int J Pharm. 1998;163:81-89.

16. Sintov AC, Botner S. Transdermal drug delivery using microemulsion and aqueous systems: Influence of skin storage conditions on the in vitro permeability of diclofenac from aqueous vehicle systems. Int $J$ Pharm. 2006;311:55-62.

17. Hasse A, Keipert S. Development and characterization of microemulsions for ocular application. Eur J Pharm Biopharm. 1997;43:179-183.

18. Szebeni J, Alving CR, Savay S, et al. Formation of complementactivating particles in aqueous solutions of Taxol: possible role in hypersensitivity reactions. Int J Immunopharmacol. 2001;1:7212-7351.

19. Lawrence MJ, Rees GD. Microemulsion - based media as novel drug delivery systems. Adv Drug Deliv Rev. 2000;45:89-121.

20. Landfester K, Willert M, Antonietti M. Preparation of polymer particles in nonaqueous direct and inverse miniemulsions. J Macromolecules. 2000;33:23702-23761.

21. Mou DS, Chen HB, Du DR, et al. Hydrogel-thickened nanoemulsion system for topical delivery of lipophilic drugs. Int J Pharm. 2008;353: $270-276$.

22. Sonneville AO, Simonnet JT, L'Alloret F. Nanoemulsions: a new vehicle for skincare products. Adv Colloid Interface Sci. 2004;108:145-149.

23. Tian QP, Li P, Wang L. Preparation of amphotericin B microemulsion and its transdermal absorption. Chin Pharm J. 2009;44:283-287.

24. Zhang Y, inventor; Hebei University of Science and Technology, assignee. Clean preparation for $(E)$-3,5-dihydroxy-4-isopropylstilbene. Chinese patent CN 101648 851. 2009 Sep 3.

25. Tadros TF, Izquierdo P, Esquena J, et al. Formation and stability of nano-emulsions. Adv Colloid Interface Sci. 2004;108:303-318.

26. Anton N, Vandamme TF. The universality of low-energy nanoemulsification. Int J Pharm. 2009;377:142-147.

27. Vandamme TF, Anton N. Low-energy nanoemulsifcation to design veterinary controlled drug delivery devices. Int J Nanomedicine. 2010;5: 867-873.

28. Uson N, Garcia MJ, Solans C. Formation of water-in-oil (W/O) nanoemulsions in a water/mixed non-ionic surfactant/oil systems prepared by a low-energy emulsification method. Colloids and Surfaces. A Physicochemical and Engineering Aspects. 2004;250:4152-4211.
29. Pan GL, Jia XB, Wei HH. Comparison among several preparation methods for pseudo-ternary phase diagrams of pharmaceutical microemulsions. J Chin Pharm. 2006;17:21-23.

30. Alicia M, Isabel S, González C, et al. Influence of the phase behavior on the properties of ionic nanoemulsions prepared by the phase inversion composition method. J Colloid Interface Sci. 2008;327:433-439.

31. Izquierdo P, Esquena J, Tadros TF, et al. Formation and stability of nano-emulsions prepared using the phase inversion temperature method. Langmuir. 2002;18:262-301.

32. Izquierdo P, Feng J, Esquena J, et al. The influence of surfactant mixing ratio on nanoemulsion formation by the PIT method. J Colloid Interface Sci. 2005;285:3882-3941.

33. Usawadee S, Onanong N, Napaporn U, et al. Characterization and mosquito repellent activity of citronella oil nanoemulsion. Int J Pharm. 2009;372:105-111.

34. Izquierdo P, Feng J, Esquena J, et al. The influence of surfactant mixing ratio on nano-emulsion formation by the PIT method. $J$ Colloid Interface Sci. 2005;28:823-941.

35. Jiang WJ, Xu QW. Preparation and preliminary stability evaluation of cyclosporine A ocular microemulsion. Chin J Pharm. 2007;38: $351-353$.

36. Almeida CP, Vital CG, Contente TC, et al. Modifcation of composition of a nanoemulsion with different cholesteryl ester molecular species: effects on stability, peroxidation, and cell uptake. Int J Nanomedicine. 2010;5:679-686.

37. Chinese State food and drug adminastration. Chinese Pharmacopoeia, 2010 ed. Appendix XIX c, Appendix VII G. 2010.

38. Bali V, Ali M, Ali J. Study of surfactant combinations and development of a novel nanoemulsion for minimising variations in bioavailability of ezetimibe. Colloids and Surfaces B. Biointerfaces. 2010;76:410-420.

39. Porter $\mathrm{CJH}$, Pouton $\mathrm{CW}$, Cuine JF, et al. Enhancing intestinal drug solubilisation using lipid-based delivery systems. Adv Drug Deliv Rev. 2008;60:673-691.
International Journal of Nanomedicine

\section{Publish your work in this journal}

The International Journal of Nanomedicine is an international, peerreviewed journal focusing on the application of nanotechnology in diagnostics, therapeutics, and drug delivery systems throughout the biomedical field. This journal is indexed on PubMed Central,

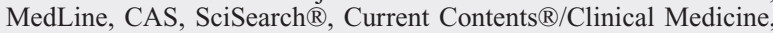

\section{Dovepress}

Journal Citation Reports/Science Edition, EMBase, Scopus and the Elsevier Bibliographic databases. The manuscript management system is completely online and includes a very quick and fair peer-review system, which is all easy to use. Visit http://www.dovepress.com/ testimonials.php to read real quotes from published authors. 